\title{
SHARP ESTIMATES FOR BLOWING DOWN FUNCTIONS IN A DENJOY-CARLEMAN CLASS
}

\author{
ANDRÉ BELOTTO DA SILVA, EDWARD BIERSTONE, AND AVNER KIRO
}

\begin{abstract}
If $F$ is a $C^{\infty}$ function whose composition $F \circ \sigma$ with a blowing-up $\sigma$ belongs to a Denjoy-Carleman class $C_{M}$, then $F$, in general, belongs to a larger class $C_{M^{(2)}}$; i.e., there is a loss of regularity. We show that this loss of regularity is sharp. In particular, loss of regularity of Denjoy-Carleman classes is intrinsic to arguments involving resolution of singularities.
\end{abstract}

\section{INTRODUCTION}

Quasianalytic Denjoy-Carleman classes $C_{M}$ go back to E. Borel [1] and were characterized (following questions of Hadamard that arose from work of Holmgren on the heat equation) by the Denjoy-Carleman theorem [15, 13. We recall that $C_{M}$ is a class of $C^{\infty}$ functions with bounds on derivatives determined by a logarithmically convex sequence $M=\left(M_{k}\right)_{k \in \mathbb{N}}$. Quasianalytic classes arise in model theory as the classes of $C^{\infty}$ functions that are definable in a given polynomiallybounded o-minimal structure [20, 25]. Relevant background on Denjoy-Carleman and quasianalytic classes is presented in Section 2 below.

Given a (log convex) sequence $M=\left(M_{k}\right)_{k \in \mathbb{N}}$ and a positive integer $p$, we denote by $M^{(p)}$ the shifted sequence $\left(M_{p k}\right)_{k \in \mathbb{N}}$, and by $M^{p}$ the sequence of $p$ th powers $\left(M_{k}^{p}\right)_{k \in \mathbb{N}}$. In general, $C_{M} \subseteq C_{M^{p}} \subseteq C_{M^{(p)}}$; moreover, $C_{M}=C_{M^{(p)}}$ if and only if $C_{M}$ is the class of analytic functions. We recall that the shifted class $C_{M^{(2)}}$ is the smallest Denjoy-Carleman class containing all $g \in \mathcal{C}^{\infty}([0, \infty))$ such that $g\left(t^{2}\right) \in C_{M}(\mathbb{R})[22$, Rmk. 6.2], cf. [12].

The goal of this paper is to extend this result to blowings-up. We can express the blowing up of the origin in the plane $\mathbb{R}^{2}$ using polar coordinates, as the mapping $\sigma: \mathbb{R}^{2} \rightarrow \mathbb{R}^{2}$ given by

$$
\sigma(r, \theta)=(r \cos \theta, r \sin \theta)
$$

(the universal covering of the standard blowing-up). If $F \in C^{\infty}\left(\mathbb{R}^{2}\right)$ is a function such that $F \circ \sigma \in C_{M}\left(\mathbb{R}^{2}\right)$, then $F \in C_{M^{(2)}}\left(\mathbb{R}^{2}\right)$, by [3, Lemma 3.4]. We will show that this estimate is sharp.

2010 Mathematics Subject Classification. Primary 26E10, 32S45; Secondary 30D60, 58C25.

Key words and phrases. Denjoy-Carleman class, quasianalytic class, blowing-up, resolution of singularities, loss of regularity, power substitution.

Research supported by NSERC Discovery Grant RGPIN-2017-06537 (Bierstone).

Research supported in part by ERC Advanced Grant 692616, ISF Grant 382/15, and by the European Research Council (ERC) under the European Union's Horizon 2020 research and innovation programme, grant agreement No 802107 (Kiro).

The authors gratefully acknowledge the referee's careful review and important comments. 
Theorem 1.1 (Sharp estimate for blowing up). Let $C_{M}$ be a Denjoy-Carleman class closed under differentiation, such that $C_{M^{2}}=C_{M^{(2)}}$. Then, for every DenjoyCarleman class $C_{N} \subsetneq C_{M^{(2)}}$, there exists $F \in C_{M^{(2)}}\left(\mathbb{R}^{2}\right) \backslash C_{N}\left(\mathbb{R}^{2}\right)$ such that $F \circ \sigma \in$ $C_{M}\left(\mathbb{R}^{2}\right)$.

Moreover, under the hypothesis that the class $C_{M}$ is quasianalytic, our techniques provide the following result.

Theorem 1.2 (Quasianalytic estimate for blowing up). Let $C_{M}$ be a quasianalytic Denjoy-Carleman class closed under differentiation. For every Denjoy-Carleman class $C_{N} \subsetneq C_{M^{(2)}}$ such that $\lim \left(N_{k} / M_{2 k}\right)^{1 / k}=0$, there exists $F \in C_{M^{(2)}}\left(\mathbb{R}^{2}\right) \backslash$ $C_{N}\left(\mathbb{R}^{2}\right)$ such that $F \circ \sigma \in C_{M}\left(\mathbb{R}^{2}\right)$.

In particular, if $C_{M}$ properly contains the analytic functions, then there exists $F \in C_{M^{(2)}}\left(\mathbb{R}^{2}\right) \backslash C_{M}\left(\mathbb{R}^{2}\right)$ such that $F \circ \sigma \in C_{M}\left(\mathbb{R}^{2}\right)$.

Note that, if $F \circ \sigma \in C_{M}$, then $F$ restricts to a function of class $C_{M}$ on any nonsingular $C_{M}$ curve (since $\sigma$ restricts to an isomorphism from a lifting of any such curve); in particular, $F$ is formally of class $C_{M}$ at every point. Therefore the loss of regularity (i.e., the fact that $F$ belongs to the Denjoy-Carleman class $C_{M^{2}}$ or $C_{M^{(2)}}$ instead of $C_{M}$, according to Theorems 1.1 or 1.2) is intrinsically a phenomenon of two or more variables - it cannot be observed by sampling the function on a nonsingular curve. An earlier example of a function of class $C^{\infty}$ (as opposed to Denjoy-Carleman) of more then one variable, which is not in $C_{M}$, although its restriction to any nonsingular $C_{M}$ curve is of class $C_{M}$, was constructed by Jaffe [17. In contrast, all explicit constructions of $C_{M}$ functions of which we are aware (e.g., 1, 6, 11, 13, 17, 19, 21, 22, 23]; cf. the historical survey 9]), are either one variable constructions or of the form $h_{1} \circ h_{2}$, where $h_{1}$ is a one variable $C_{M}$ function and $h_{2}$ is real-analytic.

The hypothesis that $C_{M^{(2)}}=C_{M^{2}}$ in Theorem 1.1 is mild in the sense that standard examples of quasianalytic Denjoy-Carleman classes usually satisfy this hypothesis (e.g., $M_{k}=\log (k)^{k}, M_{k}=\log (\log (k))^{k}$, etc.); moreover, in quasianlaytic classes, the condition is verified for "almost every" $k \in \mathbb{N}$ (see Lemma 2.5 for a precise result). It is nevertheless possible to construct examples of quasianalytic classes where the hypothesis is not satisfied.

Proposition 1.3 (Classes such that $C_{M^{2}} \subsetneq C_{M^{(2)}}$ ). There is a Denjoy-Carleman class $C_{M}$ closed under differentiation, such that $C_{M^{(2)}}$ is quasianalytic and $C_{M^{2}} \subsetneq$ $C_{M^{(2)}}$.

The paper is organized as follows. In Section 2, we provide all necessary background on quasianalytic Denjoy-Carleman classes $C_{M}$, including new results about shifted classes (Lemmas 2.2 and 2.5). In Section 3, we explicitly construct the main examples and we prove Theorems 1.1 and 1.2 . The construction relies on two preliminary steps (see $\S ₫ 3.2,3.3)$, where we provide tight estimates for the "bricks" and "building blocks" of the main construction. The main example is constructed in $\$ 3.4$ and Theorems 1.1 and 1.2 are proved in $\$ 3.5$ Finally, we prove Proposition 1.3 in Section 4 .

1.1. Algebraic properties of quasianalytic classes. A Denjoy-Carleman class $C_{M}$ which is closed under differentiation and quasianalytic admits resolution of singularities, according to [7, 8]. Resolution of singularities is a powerful technique which, roughly speaking, associates to a function $f \in C_{M}(U)$ a finite composite of 
blowings-up $\sigma: V \rightarrow U$ such that $f \circ \sigma$ is everywhere locally a monomial times a unit. This technique was explored in the last twenty years in order to study geometric and algebraic properties of quasianalytic classes [8, 25, 22, 24, 3, 5, 4,

Solutions of problems on Denjoy-Carleman classes $C_{M}$ using resolution of singularities, in general lead to loss of regularity, in the sense that a problem involving data of class $C_{M}$ has solutions in a shifted class $C_{M^{(p)}}$, for certain $p \in \mathbb{N}[3,5$. Theorem 1.1 shows that loss of regularity is an essentially unavoidable limitation of the technique of resolution of singularities. It seems important, therefore, to understand whether loss of regularity is a limitation only of the technique, or is intrinsic to geometric questions on Denjoy-Carleman classes.

For example, resolution of singularities is used in [3, Proposition 4.9] to show that that principal ideals in a local ring of functions in a quasianalytic DenjoyCarleman class $C_{M}$ are closed, modulo loss of regularity; i.e., given $f, g \in C_{M}(U)$, where $U$ is a neighbourhood of $0 \in \mathbb{R}^{n}$, if $f$ formally divides $g$ at 0 , then there is a function $h \in C_{M^{(p)}}(V)$ in a neighbourhood $V$ of 0 , such that $g=f \cdot h$, where $p \in \mathbb{N}$ depends only on $f$. If loss of regularity were necessary in this problem, this would imply that local rings in a quasianalytic class $C_{M}$ are not, in general, Noetherian. The question of Noetherianity in quasianaltyic geometry goes back to 14. (see also [27]).

\section{Preliminaries}

2.1. Denjoy-Carleman classes. We use standard multiindex notation: Let $\mathbb{N}$ denote the nonnegative integers. If $\alpha=\left(\alpha_{1}, \ldots, \alpha_{n}\right) \in \mathbb{N}^{n}$, we write $|\alpha|:=\alpha_{1}+\cdots+$ $\alpha_{n}, \alpha !:=\alpha_{1} ! \cdots \alpha_{n} !, x^{\alpha}:=x_{1}^{\alpha_{1}} \cdots x_{n}^{\alpha_{n}}$, and $\partial^{|\alpha|} / \partial x^{\alpha}:=\partial^{\alpha_{1}+\cdots+\alpha_{n}} / \partial x_{1}^{\alpha_{1}} \cdots \partial x_{n}^{\alpha_{n}}$. We also write $\partial^{\alpha}:=\partial^{|\alpha|} / \partial x^{\alpha}$.

Definition 2.1 (Denjoy-Carleman classes). Let $M=\left(M_{k}\right)_{k \in \mathbb{N}}$ denote a sequence of positive real numbers which is logarithmically convex; i.e., the sequence $\left(M_{k+1} / M_{k}\right)$ is nondecreasing. A Denjoy-Carleman class $C=C_{M}$ is a class of $\mathcal{C}^{\infty}$ functions determined by the following condition: A function $f \in \mathcal{C}^{\infty}(U)$ (where $U$ is open in $\mathbb{R}^{n}$ ) is of class $C_{M}$ if, for every compact subset $K$ of $U$, there exist constants $A, B>0$ such that

$$
\left|\frac{\partial^{|\alpha|} f}{\partial x^{\alpha}}\right| \leq A B^{|\alpha|} \alpha ! M_{|\alpha|}
$$

on $K$, for every $\alpha \in \mathbb{N}^{n}$.

The logarithmic convexity assumption implies that $M_{j} M_{k} \leq M_{0} M_{j+k}$, for all $j, k$, and that the sequence $\left(\left(M_{k} / M_{0}\right)^{1 / k}\right)$ is nondecreasing. The first of these conditions guarantees that $C_{M}(U)$ is a ring, and the second that $C_{M}(U)$ contains the ring $\mathcal{O}(U)$ of real-analytic functions on $U$, for every open $U \subset \mathbb{R}^{n}$. (If $M_{k}=1$, for all $k$, then $C_{M}=\mathcal{O}$.). A Denjoy-Carleman class $C_{M}$ is closed under composition, by Roumieu [26].

We will always assume that $M$ satisfies the additional assumption,

$$
\sup \left(\frac{M_{k+1}}{M_{k}}\right)^{1 / k}<\infty
$$

the latter implies that $C_{M}$ is closed under differentiation. The converse of this statement is due to $\mathrm{S}$. Mandelbrojt [19. In a Denjoy-Carleman class $C_{M}$, closure 
under differentiation is equivalent to closure under division by a coordinate; i.e., if $f \in C_{M}(U)$ and

$$
f\left(x_{1}, \ldots, x_{i-1}, a, x_{i+1}, \ldots, x_{n}\right)=0,
$$

where $a \in \mathbb{R}$, then $f(x)=\left(x_{i}-a\right) h(x)$, where $h \in C_{M}(U)$.

Finally, closure under differentiation implies closure under inverse (Komatsu [18; see [8] for a simple proof). More precisely, let $\varphi: U \rightarrow V$ denote a $C_{M}$-mapping between open subsets $U, V$ of $\mathbb{R}^{n}$. Let $a \in U$ and suppose that the Jacobian matrix $(\partial \varphi / \partial x)(a)$ is invertible. Then there are neighbourhoods $U^{\prime}$ of $a$ and $V^{\prime}$ of $b:=\varphi(a)$, and a $C_{M}$-mapping $\psi: V^{\prime} \rightarrow U^{\prime}$ such that $\psi(b)=a$ and $\psi \circ \varphi$ is the identity mapping of $U^{\prime}$.

2.2. Comparison between Denjoy-Carleman classes. The following criteria are due to Cartan and Mandelbrojt (see [19, Thm. XI]). If $C_{M}, C_{N}$ are DenjoyCarleman classes closed under differentiation, then:

(a) $C_{N}(U) \subseteq C_{M}(U)$, for all $U$, if and only if

$$
\sup _{k \in \mathbb{N}}\left(N_{k} / M_{k}\right)^{1 / k}<\infty
$$

(b) $C_{N}(U) \subsetneq C_{M}(U)$, for all $U$, if and only if

$$
\sup _{k \in \mathbb{N}}\left(N_{k} / M_{k}\right)^{1 / k}<\infty \quad \text { and } \quad \inf _{k \in \mathbb{N}}\left(N_{k} / M_{k}\right)^{1 / k}=0 .
$$

We note that for any Denjoy-Carleman class $C_{M}$, there is a function in $C_{M}((0,1))$ which is nowhere in any smaller class (by Jaffe [17, Thm. 1.1]; E. Borel constructed a function in $C_{M}((0,1))$ that is nowhere analytic [10, Chapt. 2]).

2.3. Shifted Denjoy-Carleman classes. Given $M=\left(M_{j}\right)_{j \in \mathbb{N}}$ and a positive integer $p$, let $M^{(p)}$ denote the sequence $M_{j}^{(p)}:=M_{p j}$. If $M$ is logarithmically convex, then $M^{(p)}$ is logarithmically convex:

$$
\frac{M_{k p}}{M_{(k-1) p}}=\frac{M_{k p}}{M_{k p-1}} \cdots \frac{M_{k p-p+1}}{M_{k p-p}} \leq \frac{M_{k p+p}}{M_{k p+p-1}} \cdots \frac{M_{k p+1}}{M_{k p}}=\frac{M_{(k+1) p}}{M_{k p}} .
$$

Therefore, if $C_{M}$ is a Denjoy-Carleman class, then so is $C_{M^{(p)}}$. Clearly, $C_{M} \subseteq$ $C_{M^{(p)}}$. Moreover, if $C_{M}$ satisfies assumption (2), then the same is true for $C_{M^{(p)}}$. We recall that $C_{M^{(2)}}$ is the smallest Denjoy-Carleman class containing all $g \in \mathcal{C}^{\infty}(\mathbb{R})$ such that $g\left(t^{2}\right) \in C_{M}(\mathbb{R})[22$, Rmk. 6.2].

In Lemma 2.2 following, we characterize sequences $M$ such that the shifted class $C_{M^{(2)}}$ equals $C_{M^{2}}$, where $M^{2}:=\left(M_{k}^{2}\right)_{k \in \mathbb{N}}$. By log-convexity, $C_{M^{2}} \subset C_{M^{(2)}}$. In order to obtain equality, we need the additional condition,

$$
\inf _{k \in \mathbb{N}}\left\{\left(\frac{M_{k}^{2}}{M_{2 k}}\right)^{1 / k}\right\}>0 .
$$

The latter is a regularity condition that complements log-convexity. More precisely, by log-convexity,

$$
\frac{M_{k}^{2}}{M_{2 k}}=M_{k} \cdot \frac{M_{k}}{M_{k+1}} \cdots \frac{M_{2 k-1}}{M_{2 k}} \leq M_{k}\left(\frac{M_{k}}{M_{k+1}}\right)^{k},
$$

so that

$$
\left(\frac{M_{k}^{2}}{M_{2 k}}\right)^{1 / k} \leq \frac{M_{k}^{1+1 / k}}{M_{k+1}}, \quad \text { for all } k \in \mathbb{N}
$$


The right hand side of (3) can be used to characterize sequences $M$ such that $C_{M^{2}} \subset C_{M^{(2)}}$ :

Lemma 2.2. Let $M$ be a log convex sequence with $M_{0}=1$. Then $C_{M^{2}}=C_{M^{(2)}}$ if and only if

$$
\inf _{k \in \mathbb{N}}\left\{\frac{M_{k}^{1+1 / k}}{M_{k+1}}\right\}>0
$$

Proof. The "only if" direction is immediate from inequality (3). To prove the converse, note that there exists a constant $a>0$ such that

$$
M_{k} \geq a^{k /(k+1)} \cdot M_{k+1}^{k /(k+1)}, \quad \text { for all } k \in \mathbb{N} .
$$

By applying this inequality $k$ times, it therefore follows that

$$
M_{k} \geq \prod_{j=1}^{k} a^{k /(k+j)} \cdot M_{2 k}^{1 / 2}, \quad \text { for all } k \in \mathbb{N},
$$

and, using Jensen's inequality, we get

$$
\left(\frac{M_{k}^{2}}{M_{2 k}}\right)^{1 / k} \geq \prod_{j=1}^{k} a^{2 /(k+j)} \geq \exp \left(\log \left(a^{2}\right) \sum_{j=1}^{k} \frac{1}{k+j}\right) \geq a^{8},
$$

proving the converse.

2.4. Quasianalytic Denjoy-Carleman classes. We say that a Denjoy-Carleman class $C_{M}$ is quasianalytic if is satisfies the following condition: if $f \in C_{M}(U)$ has Taylor expansion zero at $a \in U$, then $f$ is identically zero near $a$. According to the Denjoy-Carleman theorem [16, Thm. 1.3.8], the class $C_{M}$ is quasianalytic if and only if

$$
\sum_{k=0}^{\infty} \frac{M_{k}}{(k+1) M_{k+1}}=\infty \quad \text { or, equivalently, } \quad \sum_{k=0}^{\infty} \frac{1}{(k+1) M_{k}^{1 / k}}=\infty .
$$

(Equivalence of the latter two criteria follows from log-convexity of $M$.) In the following, a quasianalytic Denjoy-Carleman class $C_{M}$ is always assumed to be closed under differentiation. Quasianalytic Denjoy-Carleman classes $C_{M}$ admit resolution of singularities by sequences of blowings-up [7, 8].

For a quasianalytic class $C_{M}$, the shifted sequence $M^{(2)}$ and the squared sequence $M^{2}$ are "almost everywhere" comparable. In order to make this statement precise, we first recall the notion of density.

Definition 2.3 (Density of indices). Consider a set of positive integers $\Lambda$. For every $n \in \mathbb{N}$, let $A_{\Lambda}(n)$ denote the cardinality of the set $\{k \in \Lambda ; k \leq n\}$. The density $\delta(\Lambda)$ of the set $\Lambda$ is defined as

$$
\delta(\Lambda):=\lim _{n \rightarrow \infty} \frac{A_{\Lambda}(n)}{n} .
$$

Remark 2.4. By the Abel summation formula [2, Thm. 4.2],

$$
\sum_{k \in \Lambda, k \leq n} \frac{1}{k}=\int_{1}^{n} \frac{A_{\Lambda}(x)}{x^{2}} d x+\frac{A_{\Lambda}(n)}{n} .
$$


In particular, if $\delta(\Lambda)>0$ then, for every sufficiently large $n$,

$$
\sum_{k \in \Lambda, k \leq n} \frac{1}{k}>\frac{\delta(\Lambda)}{2} \log n
$$

Lemma 2.5 (Comparison between $C_{M^{2}}$ and $C_{M^{(2)}}$ ). Let $M$ be a log convex sequence with $M_{0}=1$, such that $C_{M}$ is quasianalytic. Then, for every $\varepsilon \in(0,1)$,

$$
\delta\left(\Lambda_{\varepsilon}\right)=0, \quad \text { where } \quad \Lambda_{\varepsilon}:=\left\{k: \in \mathbb{N}:\left(\frac{M_{k}^{2}}{M_{2 k}}\right)^{1 / 2 k}<1-\varepsilon\right\} .
$$

Proof. Assume, for the sake of contradiction, that $\Lambda_{\varepsilon}$ does not have zero density. Thus there exists a subset $\widetilde{\Lambda}_{\varepsilon} \subseteq \Lambda_{\varepsilon}$ of positive density. Now, there exists an increasing sequence $\left(\widetilde{a}_{k}\right)_{k \in \mathbb{N}} \subset \widetilde{\widetilde{\Lambda}}_{\varepsilon}$ such that the sets $\left\{k \in \mathbb{N} ; \widetilde{a}_{k+1} \leq 2^{n}\right\}$ have cardinality at least $\delta\left(\widetilde{\Lambda}_{\varepsilon}\right) 2^{n-1}$, for all $n$ sufficiently large.

This means that, for $n$ large enough, at most $1 / \delta\left(\widetilde{\Lambda}_{\varepsilon}\right)$ subsequent intervals $\left[2^{\ell-1}, 2^{\ell}\right), l=n+1, n+2, \ldots$, do not contain points of $\left(\widetilde{a}_{k}\right)$. We can, therefore, choose a subsequence $\left(a_{k}\right)_{k \in \mathbb{N}} \subset\left(\widetilde{a}_{k}\right)_{k \in \mathbb{N}}$ with the following properties: $a_{k+1} \geq 2 a_{k}$, each interval $\left[2^{\ell-1}, 2^{\ell}\right)$ contains at most one point $a_{k}$, and $\left\{k \in \mathbb{N} ; a_{k+1} \leq 2^{n}\right\}$ has cardinality at least $\delta\left(\widetilde{\Lambda}_{\varepsilon}\right)^{2} n / 4$, for $n$ large enough.

Now consider $L_{n}=M_{n}^{1 / n}$ and note that, since $L$ is increasing, for sufficiency large $n$,

$$
\frac{1}{L_{2^{n}}} \leq \prod_{a_{k+1} \leq 2^{n}} \frac{L_{a_{k}}}{L_{a_{k+1}}} \leq \prod_{a_{k+1} \leq 2^{n}} \frac{L_{a_{k}}}{L_{2 a_{k}}} \leq \prod_{a_{k+1} \leq 2^{n}}(1-\varepsilon) \leq(1-\varepsilon)^{\delta\left(\widetilde{\Lambda}_{\varepsilon}\right)^{2} n / 4} .
$$

We conclude that

$$
\sum_{n \geq 1} \frac{1}{n L_{n}}=\sum_{m \geq 0} \sum_{n=2^{m}}^{2^{m+1}-1} \frac{1}{n L_{n}} \leq \sum_{m \geq 0} \frac{1}{L_{2^{m}}} \sum_{n=2^{m}}^{2^{m+1}-1} \frac{1}{n} \leq(\log 2) \sum_{m \geq 0} \frac{1}{L_{2^{m}}}<\infty
$$

(where we use the fact that $1 / L_{2^{m}}$ is bounded by a geometric series). This contradicts the Denjoy-Carleman criterion (4) for quasianalyticity of $C_{M}$.

\section{Construction of the main function}

3.1. Ostrowski function. The Ostrowski function $\varphi_{M}(r)$ associated to the sequence $M$ is defined by

$$
\varphi_{M}(r):=\sup _{n \geq 0} \frac{r^{n+2}}{M_{n}}
$$

(see [23]). When there is no risk of confusion, we will denote $\varphi_{M}$ by $\varphi$. We recall the following well-known property of $\varphi_{M}$.

Lemma 3.1 (Property of Ostrowski function). Let $M$ be a log convex sequence with $M_{0}=1$, and consider the sequence $m_{k}:=M_{k+1} / M_{k}, k \in \mathbb{Z}_{\geq 0}$. The function $\varphi=\varphi_{M}$ satisfies the property,

$$
\frac{m_{k}^{2+k}}{\varphi\left(m_{k}\right)}=M_{k}
$$

Proof. Note that

$$
\frac{m_{k}^{2+k}}{\varphi\left(m_{j}\right)}=\inf _{n \geq 0} \frac{M_{n}}{m_{k}^{n-k}}=\inf _{n \geq 0} \frac{M_{n} \cdot M_{k}^{n-k}}{M_{k+1}^{n-k}} \leq M_{k} .
$$


By log-convexity of the sequence $\left(M_{k}\right)$ we get

$$
\frac{M_{n} \cdot M_{k}^{n-k}}{M_{k+1}^{n-k}} \geq \frac{M_{k} \cdot M_{k+1}^{n-k}}{M_{k+1}^{n-k}}=M_{k}, \quad \text { if } n>k,
$$

and

$$
\frac{M_{n} \cdot M_{k+1}^{k-n}}{M_{k}^{k-n}} \geq \frac{M_{k} \cdot M_{k}^{k-n}}{M_{k}^{k-n}}=M_{k}, \quad \text { if } n<k .
$$

It follows that (5) holds.

3.2. Brick function and a priori estimates. It is important to get tight estimates on derivatives of the "bricks" used in this work. In this section, we derive $a$ priori estimates via Cauchy estimates.

Remark 3.2 (Cauchy estimate). Let $f: U \subset \mathbb{C}^{2} \rightarrow \mathbb{C}$ be an holomorphic function. Fix a point $x \in U$, and positive real numbers $r_{1}$ and $r_{2}$ such that the bi-disk

$$
\mathbb{D}_{2}:=\left\{\left|z_{i}-x_{i}\right| \leq R_{i}, i=1,2\right\}
$$

is contained in $U$. The Cauchy estimate is given by:

$$
\left|\frac{\partial^{\alpha} f(x)}{\alpha !}\right| \leq \frac{\max _{z \in \mathbb{D}_{2}}|f(z)|}{R_{1}^{\alpha_{1}} R_{2}^{\alpha_{2}}}, \quad \text { for all } \alpha \in \mathbb{Z}_{\geq 0}^{2} .
$$

Given constants $q \geq 1, m \geq 1$ and $0<\rho<1$, we consider the following brick function:

$$
u_{q, m, \rho}(x):=\frac{\rho^{2}}{\rho^{2}+\left(x_{1}-\rho q\right)^{2}+\left(m x_{2}\right)^{2}} .
$$

We will use the following to estimate the derivatives of the brick function.

Lemma 3.3 (A priori estimate). For every $c \in \mathbb{R}_{>0}$ and $\alpha=\left(\alpha_{1}, \alpha_{2}\right) \in \mathbb{Z}_{\geq 0}^{2}$,

$$
\left|\frac{1}{\alpha !} \cdot \partial^{\alpha}\left(\frac{1}{c+x_{1}^{2}+x_{2}^{2}}\right)\right| \leq \frac{8 \cdot 8^{|\alpha|}}{\left(c+x_{1}^{2}+x_{1}^{2}\right)^{1+|\alpha| / 2}} .
$$

Proof. Consider the holomorphic function $f(z)=\left(c+z_{1}^{2}+z_{2}^{2}\right)^{-1}$ and a point $x \in \mathbb{R}^{2}$. Let $\mathbb{D}_{2}$ now denote the bi-disk

$$
\mathbb{D}_{2}:=\left\{\left(z_{1}, z_{2}\right) \in \mathbb{C}^{2}:\left|z_{i}-x_{i}\right| \leq 8^{-1} \sqrt{c+x_{1}^{2}+x_{2}^{2}}, i=1,2\right\},
$$

and note that $\left|x_{1}\right|+\left|x_{2}\right| \leq \sqrt{2} \sqrt{c+x_{1}^{2}+x_{2}^{2}}$. It follows from the triangle inequality that

$$
\begin{aligned}
\max _{z \in \mathbb{D}_{2}}|f(z)| & =\max _{z \in \mathbb{D}_{2}}\left(c+\left(z_{1}-x_{1}+x_{1}\right)^{2}+\left(z_{2}-x_{2}+x_{2}\right)^{2}\right)^{-1} \\
& \leq \max _{z \in \mathbb{D}_{2}}\left(c+x_{1}^{2}+x_{2}^{2}-2\left|x_{1}\right| \cdot\left|z_{1}-x_{1}\right|-2\left|x_{2}\right| \cdot\left|z_{2}-x_{2}\right|\right. \\
& \leq\left(8^{-1}\left(c+x_{1}^{2}+x_{2}^{2}\right)\right)^{-1}=8 f(x) .
\end{aligned}
$$

Using the Cauchy estimate (6), we get

$$
\left|\frac{\partial^{\alpha} f(x)}{\alpha !}\right| \leq \max _{z \in \mathbb{D}_{2}}|f(z)| \cdot\left(\frac{\sqrt{x_{1}^{2}+x_{2}^{2}+c}}{8}\right)^{-|\alpha|} \leq \frac{8 \cdot 8^{|\alpha|}}{\left(x_{1}^{2}+x_{2}^{2}+c\right)^{1+|\alpha| / 2}} .
$$


Remark 3.4 (A priori estimate on the brick function). It follows from Lemma 3.3 that, for every $q \geq 1, m \geq 1,0<\rho<1$ and $\alpha=\left(\alpha_{1}, \alpha_{2}\right) \in \mathbb{Z}_{\geq 0}^{2}$,

$$
\left|\frac{\partial^{\alpha} u_{q, m, \rho}(x)}{\alpha !}\right| \leq \rho^{2} \cdot m^{\alpha_{2}} \cdot 8^{|\alpha|+1} \cdot\left(\frac{u_{q, m, \rho}(x)}{\rho^{2}}\right)^{1+|\alpha| / 2} .
$$

Now let us consider the blowing-up $\sigma: \mathbb{R}^{2} \rightarrow \mathbb{R}^{2}$ (in polar coordinates),

$$
\sigma(r, \theta)=(r \cos \theta, r \sin \theta)
$$

and the composite brick function

$$
v_{q, m, \rho}(r, \theta):=u_{q, m, \rho} \circ \sigma(r, \theta)=\frac{\rho^{2}}{\rho^{2}+(r \cos \theta-\rho q)^{2}+(m \cdot r \sin \theta)^{2}} .
$$

Lemma 3.5 (A priori estimate on the blowing-up of the brick function). There is a (universal) constant $C>0$ such that for all real numbers $q \geq 1, m \geq 1,0<\rho<1$, as well as all $\alpha \in \mathbb{Z}_{\geq 0}^{2}, r \geq 0$ and $|\theta| \leq \pi$,

$$
\left|\frac{\partial^{\alpha} v_{q, m, \rho}(r, \theta)}{\alpha !}\right| \leq m^{|\alpha|}(1+q \rho)^{\alpha_{2}} \cdot C^{|\alpha|+1} .
$$

Proof. Fix $r \geq 0$ and $\theta \in[-\pi, \pi]$, and let $x_{1}=r \cos \theta-q \rho, x_{2}=m r \sin \theta$. By Taylor approximation, there exists $A>1$ such that for every complex number $w \in \mathbb{C}$ with $|w-\theta| \leq 1$, we have

$$
\begin{aligned}
|\sin \theta-\sin w| & \leq A \cdot|\theta-w|, \\
|\cos \theta-\cos w| & \leq|\sin \theta| \cdot|\theta-w|+A \cdot|\theta-w|^{2} .
\end{aligned}
$$

We now consider the bi-disc $\widetilde{\mathbb{D}}_{2}:=\left\{\left(w_{1}, w_{2}\right) \in \mathbb{C}^{2}:\left|w_{1}-r\right| \leq R_{1},\left|w_{2}-\theta\right| \leq R_{2}\right\}$, where the ratios are given by

$$
R_{1}:=\frac{(64 \rho)^{-1} \sqrt{\rho^{2}+x_{1}^{2}+x_{2}^{2}}}{|\cos \theta|+m|\sin \theta|}, \quad R_{2}:=\min \left\{1, \frac{(64 \rho)^{-1} \sqrt{\rho^{2}+x_{1}^{2}+x_{2}^{2}}}{m \cdot r \cdot A+r \cdot|\sin \theta|+\sqrt{r \cdot A}}\right\},
$$

and we provide estimates for $R_{1}, R_{2}$ and $\max _{w \in \widetilde{\mathbb{D}}_{2}}\left|v_{q, m, \rho}(z)\right|$ which allow us to conclude using Cauchy estimates. Indeed, since $m>1$ and $A>1$,

$$
\begin{aligned}
\frac{1}{R_{1}} & =64 \rho \cdot \frac{|\cos \theta|+m|\sin \theta|}{\sqrt{(r \cos \theta-q \rho)^{2}+m^{2}(r \sin \theta)^{2}+\rho^{2}}} \\
& \leq 64\left(1+\frac{\rho m|\sin \theta|}{\sqrt{(r \cos \theta-q \rho)^{2}+(r \sin \theta)^{2}+\rho^{2}}}\right) \leq 128 m \\
\frac{1}{R_{2}} & =\max \left\{1,64 \rho \cdot \frac{m \cdot r \cdot A+r \cdot|\sin \theta|+\sqrt{r \cdot A}}{\sqrt{\rho^{2}+x_{1}^{2}+x_{2}^{2}}}\right\} \\
& \leq 8^{3} \cdot A \cdot m \cdot \max \left\{1, \frac{3 \rho r}{\sqrt{\rho^{2}+x_{1}^{2}+x_{2}^{2}}}\right\}
\end{aligned}
$$


where in the last inequality we used the fact that either $r<1$ and the max is smaller than 3 , or $r \geq 1$ and $\sqrt{r} \leq r$. Since $m>1$ and $\cos \theta \leq 1$, we get

$$
\begin{aligned}
\frac{1}{R_{2}} & \leq 8^{3} \cdot A \cdot m \cdot \max \left\{1, \frac{3 \rho r}{\sqrt{\rho^{2}+(r \cdot \cos (\theta)-q \rho)^{2}+(m r \sin (\theta))^{2}}}\right\} \\
& \leq 8^{3} \cdot A \cdot m \cdot \max \left\{1, \frac{3 \rho r}{\sqrt{\rho^{2}+(r-q \rho)^{2}}}\right\} \\
& \leq 8^{4} \cdot A \cdot m \cdot(1+\rho q) .
\end{aligned}
$$

Let $z=\left(z_{1}, z_{2}\right)$ denote the complexification of $x$, i.e.,

$$
z_{1}=w_{1} \cdot \cos w_{2}-q, \quad z_{2}=m \cdot w_{1} \sin w_{2}
$$

(where $w_{1}, w_{2}, z_{1}, z_{2} \in \mathbb{C}$ ), so that, using the triangle inequality, we get

$$
\begin{aligned}
& \left|z_{1}-x_{1}\right| \leq r\left|\cos w_{2}-\cos \theta\right|+\left|w_{1}-r\right||\cos \theta|, \\
& \frac{\left|z_{2}-x_{2}\right|}{m} \leq r\left|\sin w_{2}-\sin \theta\right|+\left|w_{1}-r\right||\sin \theta| .
\end{aligned}
$$

Now, by the choices of $R_{1}$ and $R_{2}$, for all $w \in \widetilde{\mathbb{D}}_{2}$, we have

$$
\begin{aligned}
\left|z_{1}-x_{1}\right| & \leq\left|w_{1}-r\right||\cos \theta|+r\left(|\sin \theta| \cdot\left|w_{2}-\theta\right|+A \cdot\left|w_{2}-\theta\right|^{2}\right) \\
& \leq(64 \rho)^{-1} \sqrt{\rho^{2}+x_{1}^{2}+x_{2}^{2}} \\
\left|z_{2}-x_{2}\right| & \leq m \cdot\left|w_{1}-r\right||\sin \theta|+r \cdot A \cdot m \cdot\left|w_{2}-\theta\right| \\
& \leq(64 \rho)^{-1} \sqrt{\rho^{2}+x_{1}^{2}+x_{2}^{2}}
\end{aligned}
$$

which implies that, if $w \in \widetilde{\mathbb{D}}_{2}$, then $z \in \mathbb{D}_{2}$, where $\mathbb{D}_{2}$ is the bi-disc in the proof of Lemma 3.3. If follows from the estimate in Lemma 3.3 that

$$
\max _{w \in \widetilde{\mathbb{D}}_{2}}\left|v_{q, m, \rho}(z)\right| \leq \max _{z \in \mathbb{D}_{2}}\left|\left(1+z_{1}^{2}+z_{2}^{2}\right)^{-1}\right| \leq \frac{4}{1+x_{1}^{2}+x_{2}^{2}} \leq 4
$$

We conclude using Cauchy estimates, taking $C>0$ sufficiently big (for example, $\left.C=8^{4} A\right)$.

3.3. Building block and a priori estimates. We start with the existence of an important function.

Proposition 3.6 (Base function). Given a log convex sequence $M$ with $M_{0}=1$, there exists a "base function" $h \in C^{\infty}\left(\mathbb{R}^{2}\right)$ with the following properties:

(i) for every $x=\left(x_{1}, x_{2}\right) \in \mathbb{R}^{2}$ and $\alpha \in \mathbb{Z}_{\geq 0}^{2}$,

$$
\left|\partial^{\alpha} h(x)\right| \leq \frac{64 \cdot 8^{|\alpha|+1} \alpha !}{\left(1+x_{1}^{2}+x_{2}^{2}\right)^{1+|\alpha| / 2}} \cdot M_{\alpha_{2}},
$$

(ii) for any $n \in \mathbb{Z}_{\geq 0}$,

$$
\left|\frac{\partial^{2 n}}{\partial x_{2}^{2 n}} h(0,0)\right| \geq \frac{(2 n) !}{2^{2 n}} \cdot M_{2 n} .
$$

Proof. Let $h(x)$ denote the function

$$
h(x):=\sum_{k \geq 1} \frac{m_{k}^{2}}{2^{k} \varphi\left(m_{k}\right)} \cdot \frac{1}{x_{1}^{2}+\left(m_{k} x_{2}\right)^{2}+1},
$$


where $m_{k}=M_{k+1} / M_{k}$ and $\varphi=\varphi_{M}$ is the Ostrowski function. By Lemma 3.3 and the chain rule,

$$
\left|\partial^{\alpha} h(x)\right| \leq 64 \cdot 8^{|\alpha|+1} \alpha ! \sum_{k \geq 1} \frac{m_{k}^{2}}{2^{k} \varphi\left(m_{k}\right)} \cdot \frac{m_{k}^{\alpha_{2}}}{\left(x_{1}^{2}+\left(m_{k} x_{2}\right)^{2}+1\right)^{1+|\alpha| / 2}},
$$

for every $\alpha \in \mathbb{Z}_{\geq 0}^{2}$. It follows from the definition of $\varphi$ and the fact that $m_{k} \geq 1$ that

$$
\begin{aligned}
\left|\partial^{\alpha} h(x)\right| & \leq \frac{64 \cdot 8^{|\alpha|+1} \alpha !}{\left(x_{1}^{2}+x_{2}^{2}+1\right)^{1+|\alpha| / 2}} \sum_{k \geq 1} \frac{m_{k}^{2+\alpha_{2}}}{2^{k} \varphi\left(m_{k}\right)} \\
& \leq \frac{64 \cdot 8^{|\alpha|+1} \alpha !}{\left(x_{1}^{2}+x_{2}^{2}+1\right)^{1+|\alpha| / 2}} M_{\alpha_{2}},
\end{aligned}
$$

proving the upper-bound estimate (i). Next, from the Taylor expansion of $(1+z)^{-1}$, we get

$$
\frac{\partial^{2 n}}{\partial x_{2}^{2 n}} h(0,0)=(-1)^{n}(2 n) ! \sum_{k \geq 1} \frac{1}{2^{k}} \cdot \frac{m_{k}^{2 n+2}}{\varphi\left(m_{k}\right)},
$$

and, using Lemma 3.1, we conclude that

$$
\left|\frac{\partial^{2 n}}{\partial x_{2}^{2 n}} h(0,0)\right|=(2 n) !\left|\sum_{k \geq 1} \frac{1}{2^{k}} \cdot \frac{m_{k}^{2 n+2}}{\varphi\left(m_{k}\right)}\right| \geq \frac{(2 n) !}{2^{2 n}} \cdot \frac{m_{2 n}^{2 n+2}}{\varphi\left(m_{2 n}\right)}=\frac{(2 n) !}{2^{2 n}} \cdot M_{2 n},
$$

proving the lower bound estimate (ii).

Now, let $\rho \in(0,1)$ and let $q=(q, 0) \in \mathbb{R}^{2}$. We consider the function

$$
f_{q, \rho}(x):=h\left(\frac{x}{\rho}-q\right),
$$

and we denote by $p=(\rho \cdot q, 0)$ the associated centre point.

Remark 3.7 (A priori estimates on the building block). It follows from Proposition 3.6 and the chain rule, that for every $\rho>0$ and $q \geq 1$, the function $f_{q, \rho}(x)$ satisfies the following estimates:

(i) for every $x \in \mathbb{R}^{2}$ and $\alpha \in \mathbb{Z}_{\geq 0}^{2}$,

$$
\left|\partial^{\alpha} f_{q, \rho}(x)\right| \leq \frac{64 \rho^{2} \cdot 8^{|\alpha|+1} \alpha ! M_{\alpha_{2}}}{\left(\|x-q \rho\|^{2}+\rho^{2}\right)^{1+|\alpha| / 2}}
$$

(ii) for every $n \in \mathbb{Z}_{\geq 0}$,

$$
\left|\partial_{x_{2}}^{2 n} f_{q, \rho}(p)\right| \geq \frac{(2 n) ! \cdot M_{2 n}}{4^{n} \cdot \rho^{2 n}}
$$

Now, let us consider the blowing-up $\sigma: \mathbb{R}^{2} \rightarrow \mathbb{R}^{2}$ (in polar coordinates),

$$
\sigma(r, \theta)=(r \cos \theta, r \sin \theta) .
$$

Set $g_{\rho, q}:=f_{\rho, q} \circ \sigma(r, \theta)$. We summarize the main properties of $g_{\rho, q}$ :

Lemma 3.8 (A priori estimate on the blowing-up of the building block). Let $M=\left(M_{n}\right)$ denote a log convex sequence starting with $M_{0}=M_{1}=1$. There is a universal constant $C>0$ such that, for every $\rho \in(0,1), q \geq 1, \alpha \in \mathbb{Z}_{\geq 0}^{2}, r>0$ and $\theta \in[-\pi, \pi]$

$$
\left|\partial^{\alpha} g_{q, \rho}(r, \theta)\right| \leq C^{|\alpha|+1} \cdot(1+q \rho)^{\alpha_{2}} \cdot \alpha ! \cdot M_{|\alpha|}
$$


Proof. From the definitions of $h$ and $f_{q, \rho}$, we obtain

$$
\begin{aligned}
g_{q, \rho}(r, \theta) & =\sum_{k \geq 0} \frac{m_{k}^{2}}{2^{k} \varphi\left(m_{k}\right)} \cdot \frac{\rho^{2}}{(r \cos \theta-q \rho)^{2}+\left(m_{k} r \sin \theta\right)^{2}+\rho^{2}} \\
& =\sum_{k \geq 0} \frac{m_{k}^{2}}{2^{k} \varphi\left(m_{k}\right)} v_{q, m_{k}, \rho}(r, \theta) .
\end{aligned}
$$

where $v_{q, m_{k}, \rho}(r, \theta)=u_{q, m_{k}, \rho} \circ \sigma$ is the brick function. Now, by Lemma 3.5 with $m=m_{k}$, we get

$$
\left|\partial^{\alpha} g_{q, \rho}\right| \leq C^{|\alpha|+1} \alpha ! \cdot(1+q \rho)^{\alpha_{2}} \sum_{k \geq 0} \frac{m_{k}^{2}}{2^{k} \varphi\left(m_{k}\right)} m_{k}^{|\alpha|},
$$

and the result follows from the definition of $\varphi$.

3.4. A flat construction. Let $M$ be an increasing log convex sequence such that $M_{0}=1$. Let $E:[0, \infty) \rightarrow[0,1)$ denote an increasing continuous function such that

$$
E(0)=0 \quad \text { and } \quad \lim _{r \rightarrow 0^{+}} \frac{E(r)}{r}=\infty .
$$

Consider the sequences

$$
\rho_{n}:=M_{n} / M_{n+1}, \quad q_{n}:=\left(E\left(\rho_{n}\right) / \rho_{n}, 0\right) .
$$

Note that, whenever the class $C_{M}$ properly contains the class of analytic functions, the sequence $\left(\rho_{n}\right)$ (which is non-increasing) tends to zero. Let $\Lambda \subset 2 \mathbb{N}$ be an unbounded set of indices such that $q_{\lambda}>1$, for every $\lambda \in \Lambda$. We denote by $\Gamma$ the data $\left(\Lambda, \rho_{n}, q_{n}\right)$, and we consider the function

$$
F_{\Gamma}(x):=\sum_{\lambda \in \Lambda} \frac{f_{q_{\lambda}, \rho_{\lambda}}(x)}{\varphi\left(\rho_{\lambda}^{-1}\right) 2^{\lambda}} .
$$

We will provide conditions on the data $\Gamma$ in terms of the asymptotic behaviour of the sequence

$$
\delta_{\lambda}:=\operatorname{dist}\left(q_{\lambda} \rho_{\lambda},\left\{q_{\lambda^{\prime}} \rho_{\lambda^{\prime}}\right\}_{\lambda^{\prime} \in \Lambda \backslash\{\lambda\}}\right)=\operatorname{dist}\left(E\left(\rho_{\lambda}\right),\left\{E\left(\rho_{\lambda^{\prime}}\right)\right\}_{\lambda^{\prime} \in \Lambda \backslash\{\lambda\}}\right) .
$$

Lemma 3.9 (Regularity of $F_{\Gamma}$ ). The function $F_{\Gamma}$ belongs to $C_{M^{2}}\left(\mathbb{R}^{2}\right)$.

Proof. By the definition of $\varphi$ and Remark 3.7, if $x \in \mathbb{R}^{2}$, then

$$
\begin{aligned}
\left|\sum_{\lambda \in \Lambda} \frac{\partial^{\alpha} f_{q_{\lambda}, \rho_{\lambda}}(x)}{2^{\lambda} \varphi\left(\rho_{\lambda}^{-1}\right)}\right| & \leq 64 \cdot 8^{|\alpha|+1} \alpha ! M_{|\alpha|} \sum_{\lambda \in \Lambda} \frac{\rho_{\lambda}^{2}}{2^{\lambda} \varphi\left(\rho_{\lambda}^{-1}\right) \rho_{\lambda}^{|\alpha|+2}} \\
& \leq 8^{|\alpha|+3} \alpha ! M_{|\alpha|} M_{|\alpha|}<\infty .
\end{aligned}
$$

It follows from the Weierstrass M-test that $F_{\Gamma}$ is $C^{\infty}$ at $\mathbb{R}^{2}$, and the bounds above guarantee that it is in $C_{M^{2}}$.

Now, consider the blowing-up $\sigma(r, \theta)=(r \cos \theta, r \sin \theta)$, and set $G_{\Gamma}:=F_{\Gamma} \circ \sigma(r, \theta)$. We obtain the following estimates.

Lemma 3.10 (Estimates of $F_{\Gamma}$ after blowing up). The function $G_{\Gamma}$ belongs to $C_{M}\left(\mathbb{R}^{2}\right)$. 
Proof. From the definitions of $F_{\Gamma}$ and $G_{\Gamma}$, we get

$$
G_{\Gamma}(r, \theta)=\sum_{\lambda \in \Lambda} \frac{g_{q_{\lambda}, \rho_{\lambda}}(r, \theta)}{2^{\lambda} \varphi\left(\rho_{\lambda}^{-1}\right)}
$$

so it follows from Lemma 3.8 that

$$
\left|\partial^{\alpha} G_{\Gamma}(r, \theta)\right| \leq C^{|\alpha|+1} \alpha ! M_{|\alpha|} \sum_{\lambda \in \Lambda} \frac{1}{2^{\lambda} \varphi\left(\rho_{\lambda}^{-1}\right)}\left(1+q_{\lambda} \rho_{\lambda}\right)^{\alpha_{2}} .
$$

Since $q_{\lambda} \rho_{\lambda}=E\left(\rho_{\lambda}\right)$, which is bounded by 1 , and $\varphi\left(\rho_{\lambda}^{-1}\right) \geq 1$ (because $\rho_{\lambda}<1$ ), we obtain

$$
\left|\partial^{\alpha} G_{R}(r, \theta)\right| \leq(2 C)^{|\alpha|+1} \alpha ! M_{|\alpha|} .
$$

Lemma 3.11 (Lower estimates on $F_{\Gamma}$ ). Suppose that the class $C_{M}$ is closed under differentiation and properly contains the analytic functions. Then there is a constant $B>0$ (depending only on $M$ ) which satisfies the following property: Assume there exists $\lambda_{0} \in \Lambda$ such that $\delta_{\lambda} \geq B M_{\lambda}^{-1 / \lambda}$ when $\lambda \geq \lambda_{0}$ and $\lambda \in \Lambda \subset 2 \mathbb{N}$. Then there exists an infinite sequence of points $x_{\lambda} \rightarrow 0$ and a constant $\epsilon>0$ such that

$$
\left|\partial_{x_{2}}^{\lambda} F_{\Gamma}\left(x_{\lambda}\right)\right| \geq \frac{\epsilon^{\lambda} \lambda ! M_{\lambda} M_{\lambda}}{4^{\lambda}}, \quad \text { for } \lambda \geq \lambda_{0} \text { and } \lambda \in \Lambda \subset 2 \mathbb{N} .
$$

Proof. Consider the sequence of points $x_{n}=\rho_{n} q_{n}$, which converges to the origin since $\left(\rho_{n}\right)$ tends to zero. Note that

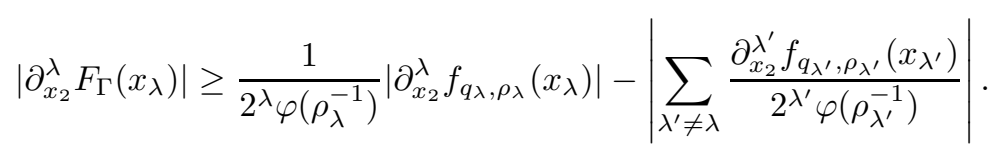

If follows from Remark 3.7 and Lemma 3.1 that

$$
\begin{aligned}
\left|\partial_{x_{2}}^{\lambda} F_{\Gamma}\left(x_{\lambda}\right)\right| & \geq \lambda ! \frac{M_{\lambda}}{\left(2 \rho_{\lambda}\right)^{\lambda} \varphi\left(\rho_{\lambda}^{-1}\right)}-\frac{\lambda ! M_{\lambda} 8^{\lambda+3}}{\delta_{\lambda}^{\lambda}} \sum_{\lambda^{\prime} \neq \lambda} \frac{1}{2^{\lambda^{\prime}}} \\
& \geq \frac{\lambda ! \rho_{\lambda}^{2} M_{\lambda} M_{\lambda}}{2^{\lambda}}-\frac{\lambda ! M_{\lambda} 8^{\lambda+3}}{\delta_{\lambda}^{\lambda}} .
\end{aligned}
$$

Now, since the class is closed under differentiation, it follows from the criterion (2) that there exists $\epsilon \in(0,1]$ such that $\rho_{\lambda}^{2} M_{\lambda} M_{\lambda} \geq \epsilon^{\lambda} M_{\lambda} M_{\lambda}$. Let $B=8^{5} \epsilon^{-1}$. Under the hypothesis of the lemma, for $\lambda \geq \lambda_{0}$,

$$
\left|\partial_{x_{2}}^{\lambda} F_{\Gamma}\left(x_{\lambda}\right)\right| \geq \frac{\epsilon^{\lambda} \lambda ! M_{\lambda} M_{\lambda}}{2^{\lambda}}-\frac{\epsilon^{\lambda} \lambda ! M_{\lambda} M_{\lambda}}{8^{\lambda}} \geq \frac{\epsilon^{\lambda} \lambda ! M_{\lambda} M_{\lambda}}{4^{\lambda}} .
$$

3.5. Proofs of the main Theorems 1.1 and 1.2. The main technical result of this section is the following lemma.

Lemma 3.12. Let $M$ denote a log convex sequence with $M_{0}=1$, such that $C_{M}$ is a Denjoy-Carleman class closed under differentiation, which properly contains the class of analytic functions. Let $\Lambda^{\prime} \subset \mathbb{N}$ be an infinite set of indices such that

$$
\inf _{k \in \Lambda^{\prime}}\left\{\left(\frac{M_{k}^{2}}{M_{2 k}}\right)^{1 / k}\right\}>0
$$


Then there exist an infinite subset $\Lambda \subset \Lambda^{\prime}$, a constant $\mathcal{K} \in \mathbb{R}_{>0}$, a sequence of points $\left(x_{\lambda}\right)_{\lambda \in \Lambda} \in \mathbb{R}^{2}$ tending to $(0,0)$, and a function $F \in C_{M^{(2)}}\left(\mathbb{R}^{2}\right)$, such that $F \circ \sigma \in C_{M}\left(\mathbb{R}^{2}\right)$ and

$$
\left|\partial_{x_{2}}^{\lambda} F\left(x_{\lambda}\right)\right| \geq \mathcal{K}^{\lambda} \lambda ! M_{2 \lambda}, \quad \text { for all } \lambda \in \Lambda .
$$

Proof. Let $E$ denote a function as in (9), and let $\rho_{n}$ and $q_{n}$ denote the sequences given by (10). Let $B>0$ be the constant (depending only on $M$ ) given by Lemma 3.11. The proof of the lemma is divided into two steps:

Step I. Suppose that $\Lambda^{\prime} \cap 2 \mathbb{N}$ is an infinite set. We construct $\Lambda$ satisfying the assumption in Lemma 3.11, By the hypothesis above and equation (3),

$$
\xi:=\inf _{\lambda \in \Lambda^{\prime}}\left\{\frac{\left(M_{\lambda}\right)^{1+1 / \lambda}}{M_{\lambda+1}}\right\}>0, \text { and } \inf _{\lambda \in \Lambda^{\prime}}\left\{\left(\frac{M_{\lambda}^{2}}{M_{2 \lambda}}\right)^{1 / \lambda}\right\}>0 .
$$

Since the sequence $\left(\rho_{n}\right)_{n \geq 1}$ is non-increasing and tends to zero, we can choose infinite $\Lambda \subset \Lambda^{\prime}$ sparse enough that $E\left(\rho_{\lambda}\right)>2 E\left(\rho_{\lambda^{\prime}}\right)$, for any $\lambda, \lambda^{\prime} \in \Lambda$ such that $\lambda<\lambda^{\prime}$. We fix such $\Lambda$, and will prove that it satisfies the assumption in Lemma3.11. Indeed, for fixed $\lambda \in \Lambda$, let $\lambda_{-}$and $\lambda_{+}$denote the smaller and larger neighbours of $\lambda$ (respectively); i.e.,

$$
\lambda_{-}:=\max (\Lambda \cap(0, \lambda)), \quad \lambda_{+}:=\min (\Lambda \cap(\lambda,+\infty)) .
$$

Let

$$
\delta_{\lambda}:=\min \left\{E\left(\rho_{\lambda_{-}}\right)-E\left(\rho_{\lambda}\right), E\left(\rho_{\lambda}\right)-E\left(\rho_{\lambda_{+}}\right)\right\} .
$$

Since $\left(\rho_{n}\right)_{n \geq 1}$ is non-increasing,

$$
\delta_{\lambda} \geq \frac{1}{2} \min \left\{E\left(\rho_{\lambda_{-}}\right), E\left(\rho_{\lambda}\right)\right\}=\frac{E\left(\rho_{\lambda}\right)}{2} .
$$

By (9) and the first condition in (11), there exists $\lambda_{0}$ such that

$$
\delta_{\lambda} \geq \frac{E\left(\rho_{\lambda}\right)}{2}>B \xi^{-1} \rho_{\lambda}=B \xi^{-1} \frac{M_{\lambda}}{M_{\lambda+1}} \geq \frac{B}{M_{\lambda}^{1 / \lambda}}, \quad \text { for } \lambda \geq \lambda_{0},
$$

showing that the the assumption in Lemma 3.11 is satisfied.

Finally, consider the function $F_{\Gamma}$ determined by the data $\left(\Lambda, \rho_{n}, q_{n}\right)$. By Lemmas 3.9 and 3.10, $F \in C_{M^{2}}\left(\mathbb{R}^{2}\right) \subset C_{M^{(2)}}\left(\mathbb{R}^{2}\right)$ and $F_{\Gamma} \circ \sigma \in C_{M}\left(\mathbb{R}^{2}\right)$. By Lemma 3.11 and the the second condition in (11), there exists $\epsilon>0$ and a sequence of points $x_{\lambda} \rightarrow 0$ such that

$$
\left|\partial_{y}^{\lambda} F_{\Gamma}\left(x_{\lambda}\right)\right| \geq \frac{\epsilon^{\lambda} \lambda ! M_{\lambda} M_{\lambda}}{4^{\lambda}} \geq \frac{\epsilon^{\lambda} \lambda ! M_{2 \lambda}}{8^{\lambda}}, \quad \text { for } \lambda \geq \lambda_{0},
$$

as we wanted to prove.

Step II. Suppose that $\Lambda^{\prime} \cap 2 \mathbb{N}$ is a finite set. Consider the set of indexes $\widetilde{\Lambda}^{\prime}=\{\lambda+1$ : $\left.\lambda \in \Lambda^{\prime}\right\}$ and note that $\widetilde{\Lambda}^{\prime} \cap 2 \mathbb{N}$ is infinite. It follows from the Step I that there exist an infinite subset $\widetilde{\Lambda} \subset \widetilde{\Lambda}^{\prime}$, a constant $\widetilde{\mathcal{K}} \in \mathbb{R}_{>0}$, a sequence of points $\left(x_{\widetilde{\lambda}}\right)_{\tilde{\lambda} \in \widetilde{\Lambda}} \in \mathbb{R}^{2}$, and a function $\widetilde{F} \in C_{M^{(2)}}\left(\mathbb{R}^{2}\right)$, such that $F \circ \sigma \in C_{M}\left(\mathbb{R}^{2}\right)$ and

$$
\left|\partial_{x_{2}}^{\lambda} F\left(x_{\widetilde{\lambda}}\right)\right| \geq \widetilde{\mathcal{K}}^{\lambda} \lambda ! M_{2 \lambda}, \quad \text { for all } \lambda \in \widetilde{\Lambda} \text {. }
$$

Let $\Lambda:=\{\lambda-1 ; \lambda \in \widetilde{\Lambda}\} \subset \Lambda^{\prime}$ and set $F:=\partial_{x_{2}} \widetilde{F}$. Since $C_{M^{(2)}}$ is closed under differentiation (cf. \$2.3), it follows that $F \in C_{M^{(2)}}\left(\mathbb{R}^{2}\right)$. Note that

$$
F \circ \sigma=\frac{1}{r}\left(r \sin \theta \partial_{r}(\widetilde{F} \circ \sigma)+\cos \theta \partial_{\theta}(\widetilde{F} \circ \sigma)\right) ;
$$


it follows that $F \circ \sigma \in C_{M}$, since $\widetilde{F} \circ \sigma \in C_{M}$ and $C_{M}$ is closed under differentiation and division by a monomial. Finally, note that the point $x_{\lambda}$ is well-defined for every $\lambda \in \Lambda$, and

$$
\left|\partial_{x_{2}}^{\lambda} F\left(x_{\lambda}\right)\right|=\left|\partial_{x_{2}}^{\lambda+1} \widetilde{F}\left(x_{\widetilde{\lambda}}\right)\right| \geq \widetilde{\mathcal{K}}^{\lambda+1}(\lambda+1) ! M_{2 \lambda+2} \geq \mathcal{K}^{\lambda} \lambda ! M_{2 \lambda} .
$$

for some $\mathcal{K}>0$; this completes the proof of the second step and of the lemma.

We can now prove the main theorems.

Proof of Theorem 1.1. The result is trivial when $C_{M}$ is the class of analytic functions, so suppose that $C_{M}$ properly contains the analytic function and consider $C_{N} \subsetneq C_{M^{(2)}}$. By hypothesis, $C_{M^{2}}=C_{M^{(2)}}$. By the criterion of $\$ 2.2$ (b) applied to $C_{N}$ and $C_{M^{2}}$, there exists an infinite subset $\Lambda^{\prime} \subset \mathbb{N}$ such that

$$
\lim _{k \rightarrow \infty, k \in \Lambda^{\prime}}\left\{\left(\frac{N_{k}}{M_{2 k}}\right)^{1 / k}\right\}=0 \text { and } \inf _{k \in \Lambda^{\prime}}\left\{\left(\frac{M_{k}^{2}}{M_{2 k}}\right)^{1 / k}\right\}>0 .
$$

The result follows easily from Lemma 3.12

Proof of Theorem 1.2. The result is trivial when $C_{M}$ is the class of analytic functions, so suppose that $C_{M}$ properly contains the analytic function and consider $C_{N} \subsetneq C_{M^{(2)}}$ such that $\lim _{k \rightarrow \infty}\left(N_{k} / M_{2 k}\right)^{1 / k}=0$. By Lemma 2.5. there exists an infinite subset of indices $\Lambda^{\prime} \subset \mathbb{N}$ such that

$$
\inf _{k \in \Lambda^{\prime}}\left\{\left(\frac{M_{k}^{2}}{M_{2 k}}\right)^{1 / k}\right\}>0
$$

whereas $\inf _{k \in \Lambda^{\prime}}\left\{\left(N_{k} / M_{2 k}\right)^{1 / k}\right\}=0$. The result follows easily from Lemma 3.12

\section{Proof of Proposition 1.3}

Fix a positive increasing sequence $\left(v_{k}\right)$ such that

$$
v_{k} \rightarrow \infty, \quad \frac{v_{k+1}}{v_{k}} \uparrow 1 \text { and } \quad v_{2 k} / v_{k} \uparrow \infty ;
$$

for example, we may take $v_{k}=\prod_{j \leq k}(1+1 / \sqrt{j})$, for every $k \in \mathbb{N}$. Let $\Lambda=\left(\lambda_{n}\right)$ be an increasing sequence of nonnegative even integers with $\lambda_{0}=0$, such that

$$
|\Lambda \cap 2 \cdot \Lambda|=\infty, \quad \text { and } \quad \sum_{n>0} \frac{\log \lambda_{n+1}-\log \lambda_{n}}{v_{n}^{2}}=\infty .
$$

For every $k \in \mathbb{N}$ such that $\lambda_{n} \leq k<\lambda_{n+1}$, set $L_{k}=v_{n}$. Finally, set $M_{k}=L_{k}^{k}$. Let us verify that $C_{M}$ is the class sought:

$M$ is a log convex sequence. Indeed, let $a_{k}=\log M_{k}-\log M_{k-1}$. By direct computation,

$$
\begin{array}{ll}
a_{k}=\log \left(v_{n}\right), & \text { if } \lambda_{n}<k \leq \lambda_{n} ; \\
a_{k}=\log \left(v_{n}\right)+(k-1) \log \left(\frac{v_{n}}{v_{n-1}}\right), & \text { if } k=\lambda_{n} .
\end{array}
$$

This implies that $\left(a_{k}\right)$ is an increasing sequence, and the result follows. 
$C_{M}$ is closed under differentiation. Let

$$
b_{k}=\left(\frac{M_{k+1}}{M_{k}}\right)^{1 / k}=\frac{L_{k+1}}{L_{k}} \cdot L_{k+1}^{1 / k} .
$$

It is enough to show that $b_{k}$ is bounded from above. By the inequality of arithmetic and geometric means, $b_{k} \leq\left(L_{k+1} / L_{k}\right)^{2}$, and direct computation shows that

$$
\begin{array}{ll}
\left(\frac{L_{k+1}}{L_{k}}\right)^{2}=1, & \text { if } \lambda_{n}<k+1 \leq \lambda_{n} ; \\
\left(\frac{L_{k+1}}{L_{k}}\right)^{2}=\left(\frac{v_{n}}{v_{n-1}}\right)^{2}, & \text { if } k+1=\lambda_{n},
\end{array}
$$

so there is indeed an upper bound.

$C_{M^{(2)}}$ is quasianalytic. Since $L_{n}$ is non-decreasing,

$$
\sum_{n \geq 1} \frac{1}{n L_{2 n}^{2}} \geq \sum_{n \geq 1} \frac{1}{L_{2^{n+1}}^{2}}=\sum_{n \geq 1} \sum_{\lambda_{n} \leq 2^{k+1}<\lambda_{n+1}} \frac{1}{L_{n}^{2}} \geq \sum_{n \geq 1} \frac{\log _{2} \lambda_{n+1}-\log _{2} \lambda_{n}}{v_{n}^{2}} .
$$

By assumption, the sum on the right hand side is divergent.

$C_{M^{2}} \subsetneq C_{M^{(2)}}$. Observe that

$$
\left(\frac{M_{k}^{2}}{M_{2 k}}\right)^{1 / k}=\left(\frac{L_{k}}{L_{2 k}}\right)^{2}
$$

Since $\Lambda \cap 2 \Lambda$ is an infinite and $v_{k} / v_{2 k} \rightarrow 0$, we see that zero is a partial limit of the sequence above. By construction, the partial limits of this sequence are 0 and 1. In particular,

$$
\limsup _{k \rightarrow \infty} \frac{M_{n}^{2}}{M_{2 n}} \leq 1 \text { and } \quad \liminf _{k \rightarrow \infty} \frac{M_{n}^{2}}{M_{2 n}}=0
$$

The assertion follows from the criterion $\$ 2.2(\mathrm{~b})$.

\section{REFERENCES}

[1] F. Acquistapace, F. Broglia, M. Bronshtein, A.Nicoara and N. Zobin, Failure of the Weierstrass preparation theorem in quasi-analytic Denjoy-Carleman rings, Adv. Math. 258 (2014), 397413.

[2] T. Apostol, Introduction to Analytic Number Theory, Undergraduate Texts in Mathematics, Springer-Verlag, 1976.

[3] A. Belotto da Silva, I. Biborski and E. Bierstone, Solutions of quasianalytic equations, Selecta Math. New Ser. 23 (2017), 2523-2552.

[4] A. Belotto da Silva and E. Bierstone, Monomialization of a quasianalytic morphism, arXiv 1907.09502 [math.AG], 2019.

[5] A. Belotto da Silva, E. Bierstone and M. Chow Composite quasianalytic functions Compositio Math., 154 (2018), 1960-1973.

[6] A. Beurling, The Collected Works of Arne Beurling, Vol. 1, Complex Analysis, Ed. L. Carleson, P. Malliavin, J. Neuberger and J. Wermer, Contemp. Math., Birkhäuser Boston, Boston, MA, 1989.

[7] E. Bierstone and P.D. Milman, Canonical desingularization in characteristic zero by blowing up the maximum strata of a local invariant, Invent. Math. 128 (1997), 207-302.

[8] E. Bierstone and P.D. Milman, Resolution of singularities in Denjoy-Carleman classes, Selecta Math. (N.S.) 10 (2004), 1-28.

[9] G. G. Bilodeau, The origin and early development of non-analytic infinitely differentiable functions, Archive for History of Exact Sciences. 27 (1982), 115-135. 
[10] E. Borel, Sur quelques points de la théorie des fonctions, Ann. Sci. École Norm. Sup. 12 (1895), 9-55.

[11] E. Borel, Sur la généralisation du prolongement analytique, C. R. Acad. Sci. Paris 130 (1900), $1115-1118$.

[12] L. Buhovsky, A. Kiro and S. Sodin, Power substitution in quasianalytic Carleman classes, Isr. J. Math. 235 (2020), 79-90.

[13] T. Carleman, Les Fonctions Quasi-analytiques, Collection Borel, Gauthier-Villars, Paris, 1926.

[14] C.L. Childress, Weierstrass division in quasianalytic local rings, Can. J. Math. 28 (1976), 938-953.

[15] A. Denjoy, Sur les fonctions quasi-analytiques de variable reélle, C. R. Acad. Sci. Paris 173 (1921), 3120-1322.

[16] L. Hörmander, The Analysis of Linear Partial Differential Operators I, Springer-Verlag, Berlin-Heidelberg-New York, 1983.

[17] E. Jaffe, Pathological phenomena in Denjoy-Carleman classes, Can. J. Math. 68 (2016), $88-108$.

[18] H. Komatsu, The implicit function theorem for ultradifferentiable mappings, Proc. Japan Acad. 55 (1979), 69-72.

[19] S. Mandelbrojt, Séries Adhérentes, Régularisation des Suites, Applications, Collection Borel, Gauthiers-Villars, Paris, 1952.

[20] C. Miller, Infinite differentiability in polynomially bounded o-minimal structures, Proc. Amer. Math. Soc. 123 (1995), 2551-2555.

[21] F. Nazarov, M. Sodin and A. Volberg, Lower bounds for quasianalytic functions. I. How to control smooth functions, Math. Scand. 95 (2004), 59-79.

[22] K.J. Nowak, Quantifier elimination in quasianalytic structures via non-standard analysis, Ann. Polon. Math. 114 (2015), 235-267.

[23] A. Ostrowski, Über quasianalytische Funktionen und Bestimmtheit asymptotischer Entwickleungen, Acta Math. 53 (1929),181-266.

[24] J.-P. Rolin and T. Servi, Quantifier elimination and rectilinearization theorem for generalized quasianalytic algebras, Proc. Lond. Math. Soc. (3) 110 (2015), 1207-1247.

[25] J.-P. Rolin, P. Speissegger and A. J. Wilkie, Quasianalytic Denjoy-Carleman classes and o-minimality J. Amer. Math. Soc. 16 (2003), 751-777.

[26] C. Roumieu, Ultradistributions définies sur $\mathbb{R}^{n}$ et sur certaines classes de variét'es différentiables, J. Analyse Math. 10 (1962-63), 153-192.

[27] V. Thilliez, On quasianalytic local rings, Expo. Math. 26 (2008), 1-23.

(A. Belotto da Silva) Université AiX-Marseille, Institut de Mathématiques de MarSeille (UMR CNRS 7373), Centre de Mathématiques et Informatique, 39 rue F. Joliot Curie, 13013 Marseille, France

Email address, A. Belotto da Silva: andre-ricardo.belotto-da-silva@univ-amu.fr

(E. Bierstone) University of Toronto, Department of Mathematics, 40 St. George Street, Toronto, ON, Canada M5S 2E4

Email address, E. Bierstone: bierston@math.utoronto.ca

(A. Kiro) Weizmann Institute of Science,Faculty of Mathematics and Computer Science, Department of Mathematics, Rehovot, Israel

Email address, A. Kiro: avner-ephraiem.kiro@weizmann.ac.il 